\title{
Sulopenem Etzadroxil
}

National Cancer Institute

\section{Source}

National Cancer Institute. Sulopenem Etzadroxil. NCI Thesaurus. Code C87678.

An orally available ester prodrug form of sulopenem, a thiopenem with broad-spectrum antibacterial activity ag ainst most gram-positive and gram-negative bacteria. After oral administration of sulopenem etzadroxil, the ester bond is cleaved, releasing active sulopenem. Sulopenem is not active against Pseudomonas aeruginosa. In addition, this agent is fairly stable against hydrolysis by various beta-lactamases. 